Journal for ImmunoTherapy of Cancer

\section{Histone deacetylase inhibitors valproic acid and vorinostat enhance trastuzumab-mediated antibody- dependent cell-mediated phagocytosis}

To cite: Laengle J, Kabiljo J, Hunter L, et al. Histone deacetylase inhibitors valproic acid and vorinostat enhance trastuzumab-mediated antibody-dependent cellmediated phagocytosis. Journal for ImmunoTherapy of Cancer 2020;8:e00195. doi:10.1136/ jitc-2019-000195

- Prepublication history and additional material for this paper are available online. To view these files, please visit the journal online (http://dx.doi.org/ 10.1136/jitc-2019-000195).

Accepted 01 November 2019

(C) Author(s) (or their employer(s)) 2020. Re-use permitted under CC BY-NC. No commercial re-use. See rights and permissions. Published by BMJ.

${ }^{1}$ Division of General Surgery, Department of Surgery, Comprehensive Cancer Center Vienna, Medical University of Vienna, Vienna, Austria ${ }^{2}$ Ludwig Boltzmann Institute Applied Diagnostics, Medical University of Vienna, Vienna, Austria

${ }^{3}$ Department of Pathology, Comprehensive Cancer Center Vienna, Medical University of Vienna, Vienna, Austria

Correspondence to Michael Bergmann; michael.bergmann@ meduniwien.ac.at

\section{ABSTRACT}

Background The monoclonal antibody (mAb) trastuzumab is part of the standard of care for patients with human epidermal growth factor receptor 2 (HER2)-overexpressing breast cancer. Antibody-dependent cell-mediated phagocytosis (ADCP) and cytotoxicity (ADCC) are major mechanisms of action of the mAb trastuzumab. Histone deacetylase inhibitors (HDACi), such as valproic acid (VPA) or vorinostat (SAHA), exert several immunostimulatory properties, which contribute at least in part to their anticancer effect. However, the impact of HDACi-induced immunostimulatory effects on trastuzumab-mediated antitumor immune response is not well characterized.

Methods We analyzed the ADCP and ADCC activity of peripheral blood mononuclear cells (PBMCs) from age and gender-matched healthy volunteers $(n=5)$ against HDACitreated HER2-overexpressing breast cancer cells (SKBR3), using a well-established in vitro three-color imaging flow cytometry and flow cytometry approach.

Results VPA and SAHA enhanced trastuzumab-mediated ADCP and trastuzumab-independent cytotoxicity. Mechanistically, VPA upregulated the activating antibody-binding receptor $\mathrm{Fc}$-gamma receptor $(\mathrm{Fc} \gamma \mathrm{R})$ IIA (CD32A) on monocytes (CD14+). Moreover, VPA and SAHA downregulated the anti-apoptotic protein myeloid leukemia cell differentiation 1 (MCL1) in breast cancer cells. Additionally, VPA and SAHA induced an immunogenic cell death, characterized by the exposure of calreticulin (CALR), as well as decreased the "do not eat me" signal CD47 on tumor cells.

Conclusions HDACi VPA and SAHA increase trastuzumabmediated phagocytosis and trastuzumab-independent cytotoxicity. The immunomodulatory activities of those HDACi support a rationale combined treatment approach with $\mathrm{mAb}$ for cancer treatment.

\section{BACKGROUND}

Monoclonal antibody (mAb) treatment, has evolved as a valuable approach in various types of human solid malignancies. In particular, trastuzumab, targeting the human epidermal growth factor receptor 2 (HER2), has become the standard of care treatment for women with HER2-overexpressing breast cancer. ${ }^{12}$
Trastuzumab has multiple direct and indirect anti-tumor effects, such as the inhibition of cell survival and proliferation. ${ }^{3-5}$ Several studies further highlighted antibody-dependent cellmediated phagocytosis (ADCP) and cytotoxicity (ADCC) as major mechanisms of action for most mAb, such as trastuzumab. ${ }^{67}$ Although HER2-overexpressing breast cancer patients benefit from trastuzumab treatment, response rates only range between $19 \%$ and $26 \%$ when administered as monotherapy. ${ }^{8} \mathrm{~A}$ better understanding of trastuzumab's molecular mechanisms could lead to the development of a more rational combination treatment approach, which would improve patient outcome.

In this line, histone deacetylase inhibitors (HDACi) represent a new class of targeted anti-cancer therapeutics, which have different effects, such as induction of apoptosis or autophagic cell death. ${ }^{10} 11$ These agents selectively inhibit the activity of histone deacetylases (HDACs), and thereby, alter gene transcription by unbalancing the acetylation of histones and other proteins. HDACi can reverse epigenetic abnormalities that are associated with carcinogenesis and thereby reboot intrinsic anti-tumor responses, such as cell cycle arrest and cell differentiation in vitro. ${ }^{12-14}$ Additionally, HDACi (panobinostat) have been found to engage the immune system and contribute to an effective anti-tumor response in murine models. ${ }^{15} 16$ Furthermore, cancer cell treatment with HDACi has been shown to enhance their susceptibility to natural killer (NK) cell cytotoxicity or dendritic cell (DC) phagocytosis in vivo. ${ }^{17-19}$ These immunostimulatory properties may improve the host's immune responses and clinical outcomes of immunotherapies, such as trastuzumab treatment.

It has been suggested that HDACi have additive or synergistic treatment effects in 
combination with the mAb trastuzumab in vitro. ${ }^{20-22}$ These data have been generated on cell lines in the absence of immune cells. However, murine models using xenografts indicated that the synergistic effect of the HDACi panobinostat and trastuzumab is based on the activation of NK cells, supporting the idea that HDACiinduced immunomodulation is essential for its therapeutic effect. ${ }^{15}$ The combination of trastuzumab with the HDACi vorinostat, also known as suberanilohydroxamic acid (SAHA), has even been taken into a phase I/II clinical trial for HER2-overexpressing breast cancer patients. ${ }^{23}$ Thus, despite promising preclinical and clinical data, the impact of HDACi on the trastuzumab-mediated human immune response on a functional level is currently not fully understood.

Therefore, we investigated the influence of two different HDACi, SAHA and valproic acid (VPA), on human trastuzumab-mediated ADCP and ADCC, by the use of a well-established in vitro three-color flow cytometry (FCM) approach. Strikingly, VPA and SAHA enhanced trastuzumab-mediated ADCP and trastuzumabindependent cytotoxicity. To elucidate the molecular mechanism of this synergistic effect, we evaluated the impact of molecules, which appear relevant for antibodymediated immunogenic cell death. In this line, we focused on (i) myeloid leukemia cell differentiation protein 1 (MCL1), as anti-apoptotic protein, which has been shown inhibit $\mathrm{ADCC}^{24}$ and being modulated by $\mathrm{HADCi}^{13}$; (ii) the expression of antibody-binding Fc-gamma receptor $(\mathrm{F} c \gamma \mathrm{R})$ subtypes, which either mediate activation or suppression of the respective immune cell ${ }^{25}$; (iii) CD47, a receptor, which suppresses phagocytosis and acts as a checkpoint molecule on antigen-presenting cells $^{26}$; and (iv) calreticulin (CALR), a molecules, which acts as a key molecule for immunogenic cell death. ${ }^{27}$

\section{METHODS \\ Cell line}

The human female breast adenocarcinoma cell line SKBR3 (ATCC, Manasses, Virginia, USA), which overexpresses the human epidermal growth factor receptor 2 (HER2), was maintained in RPMI 1640 Medium GlutaMAX Supplement (Thermo Fisher Scientific Inc., Waltham, Massachusetts, USA), supplemented with $10 \%$ heat-inactivated (hi) fetal calf serum (FCS; Linaris, Wertheim-Bettingen, GER). Cells were incubated at $37^{\circ} \mathrm{C}$ in a $5 \% \mathrm{CO}_{2}$ humidified environment.

\section{Drugs}

Trastuzumab (Herceptin) was provided by the institutional pharmacy (Vienna General Hospital, Medical University of Vienna). VPA (Sigma-Aldrich, Saint Louis, Missouri, USA) was dissolved in Dulbecco's phosphatebuffered saline without calcium and magnesium (DPBS -/-; Thermo Fisher Scientific Inc.). Vorinostat (Zolinza), also known as suberanilohydroxamic acid (SAHA;
Selleckchem, Munich, GER) was dissolved in dimethyl sulfoxide (DMSO; Thermo Fisher Scientific Inc.).

\section{VPA and SAHA titration}

SKBR3 cells were treated with either VPA or SAHA at different concentrations (online supplementary figure S1a, b), collected after 24 hours (h), washed with DPBS $-/-$ and subsequently stained with the FITC Annexin V Apoptosis Detection Kit I (BD Pharmingen, Franklin Lakes, New Jersey, USA), according to manufacturer's protocol. Subsequently, cells were measured by a Gallios G flow cytometer (Beckman Coulter Inc., Brea, California, USA), and further analyzed with Kaluza analysis software (V.2.1.1; Beckman Coulter Inc.). Viable cells were determined as Annexin $\mathrm{V}$ and propidium iodide (PI) double negative (Annexin V-/PI-), early apoptotic cells as Annexin V positive and PI negative (Annexin V+/ PI-) and end-stage apoptotic dead cells as Annexin V and PI double positive (Annexin V+/PI+).

\section{Isolation of peripheral blood mononuclear cells (PBMCs)}

Peripheral blood $(27 \mathrm{~mL})$ was drawn into VACUETTE EDTA tubes (Greiner Bio-One International, Kremsmuenster, Upper Austria, AUT) from sex-matched and aged-matched healthy volunteers $(n=5)$. PBMCs were immediately isolated using Ficoll-Paque gradient centrifugation (GE Healthcare Bio-Sciences, Uppsala, Sweden). PBMCs were washed twice with DPBS -/- and further resuspended in RPMI 1640 Medium GlutaMAX supplemented with $10 \%$ FCS.

\section{Target cell staining and pretreatment with VPA or SAHA}

SKBR3 cells were trypsinized (Thermo Fisher Scientific Inc.) and resuspended in DPBS -/- to a concentration of $5 \times 10^{7} / \mathrm{mL}$. The cells were further incubated with $1 \mu \mathrm{L}$ of $0.5 \mathrm{mM}$ carboxyfluorescein diacetate succinimidyl ester (CFSE; Invitrogen, Eugene, Oregon, USA) per $1 \times 10^{6}$ cells for $10 \mathrm{~min}(\mathrm{~min})$ at $37^{\circ} \mathrm{C}$ in a $5 \% \mathrm{CO}_{2}$ humidified atmosphere. Afterwards cells were washed in RPMI 1640 Medium GlutaMAX supplemented with 10\% FCS and resuspended to a concentration of $5 \times 10^{5} / \mathrm{mL}$. The cells $(200 \mu \mathrm{L})$ were further put into $5 \mathrm{~mL}$ tubes (BD Falcon) and treated either with $5 \mathrm{mM}$ VPA, $10 \mathrm{mM}$ VPA, $5 \mu \mathrm{M}$ SAHA, $10 \mu \mathrm{M}$ SAHA or left untreated for 24 hours at $37^{\circ} \mathrm{C}$ in a $5 \% \mathrm{CO}_{2}$ humidified environment.

\section{Antibody-dependent cell-mediated cytotoxicity phagocytosis (ADCP) and cytotoxicity (ADCC) assay}

ADCP and ADCC activities were measured as previously described. ${ }^{28-30}$ Briefly, freshly isolated PBMCs were resuspended in RMPI 1640 supplemented with $10 \%$ FCS to the concentration of $12.5 \times 10^{6} / \mathrm{mL}$ or $6.25 \times 10^{6} / \mathrm{mL}$. PBMCs $(200 \mu \mathrm{L})$ were added to the CFSE-labeled and HDACi-pretreated SKBR3 cells, resulting in an effector to target (E:T) ratio of 25:1 or 12.5:1 (total reaction volume $400 \mu \mathrm{L}$ ). The cells were incubated together for 2.5 hours at $37^{\circ} \mathrm{C}$ in a $5 \% \mathrm{CO}_{0}$ humidified environment in the presence or absence of trastuzumab at a concentration of $2.5 \mu \mathrm{g} / \mathrm{mL}$ (figure 1 ). 
A

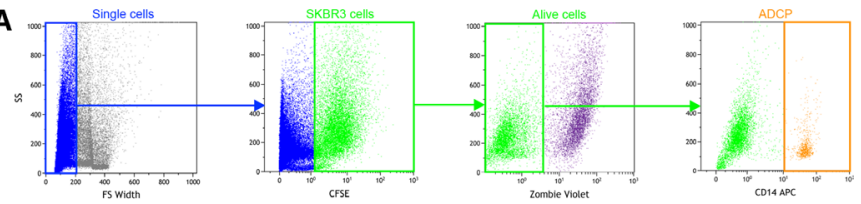

B

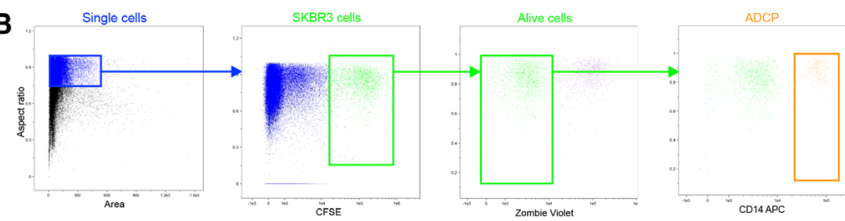

C

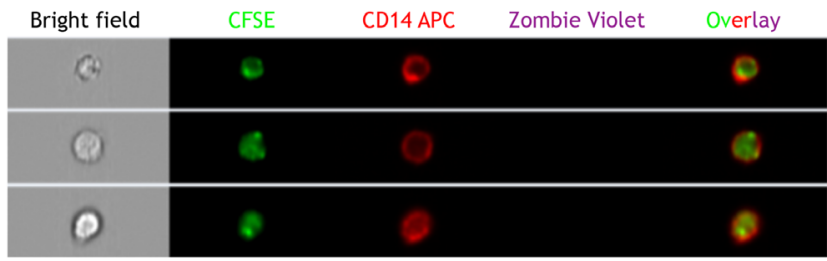

D ADCP comparison - FCM vs. IFC

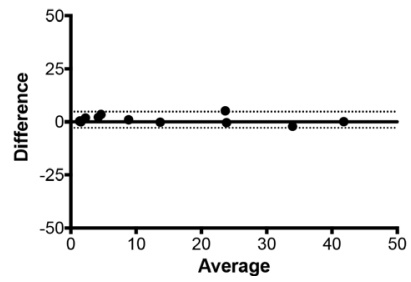

Figure 1 Measurement of ADCP with FCM and IFC is correlated with each other. ADCP/ADCC assay was performed as described in figure 1 and measured by FCM and IFC. (A) FCM gating strategy for ADCP. (B) IFC gating strategy ADCP. (C) ADCP example images obtained by IFC. (D) Bland-Altman plots show the difference (FCM-IFC), depicted on the ordinate, and the average ((FCM+IFC)/2), represented at the abscissa, of the two different measurements. Dotted lines represent the $95 \%$ limits of agreement. Each dot represents one individual experiment. Bias (0.983) and $95 \%$ limits of agreement (-2.822 to 4.789$)$. ADCC, antibody-dependent cell-mediated cytotoxicity; ADCP, antibody-dependent cell-mediated phagocytosis; APC, allophycocyanin; CFSE, carboxyfluorescein succinimidyl ester; FCM, flow cytometry; FS, forward scatter; IFC, imaging flow cytometry; SS, side scatter.

Afterwards, the cells were washed in DPBS -/and stained using Zombie Violet Fixable Viability Kit (BioLegend, San Diego, California, USA), according to the manufacturer's protocol. Next, the cells were washed with DPBS - /- supplemented with 2\% hi FCS and stained with anti-human CD14 allophycocyanin (APC) for $30 \mathrm{~min}$ at $4^{\circ} \mathrm{C}$.

Staining events were quantified by a Gallios G flow cytometer (Beckman Coulter Inc.) or Amnis ImageStreamXMark II imaging flow cytometer (IFC; Luminex Corporation, Austin, Texas, USA) and further analyzed with Kaluza Analysis Software V.2.1.1 (Beckman Coulter Inc.) or IDEAS software (Luminex Corporation).
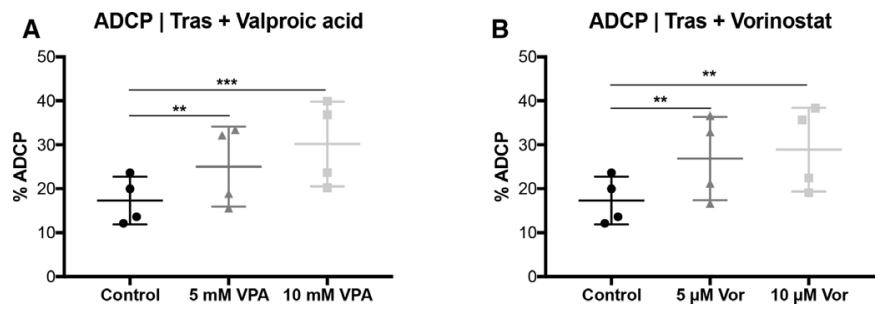

Figure 2 VPA and vorinostat enhance trastuzumabmediated ADCP. SKBR3 cells were treated with VPA or SAHA, for 24 hours, following the ADCP/ADCC assay as described in figure 1 and measured by FCM. (A) ADCP | VPA (B) ADCP | vorinostat. Results are illustrated by scatter plots. Circles, boxes and triangles illustrate individual measured values. \% ADCP is depicted on the ordinate, different treatments and corresponding concentrations are indicated on the abscissa. Mean ADCP \% is illustrated by a long horizontal line $\pm S D$ by short horizontal lines connected with a vertical line. Differences were calculated by repeated-measure one-way ANOVA and Fisher LSD post hoc test. ${ }^{* *} \mathrm{p}<0.01 ;{ }^{* \star *} \mathrm{p}<0.001$; ADCC, antibody-dependent cell-mediated cytotoxicity; ADCP, antibody-dependent cell-mediated phagocytosis; ANOVA, analysis of variance; FCM, flow cytometry; SAHA, suberanilohydroxamic acid; Tras, trastuzumab; VPA, valproic acid.

CFSE and CD14 APC double-positive cells (CFSE+/ $\mathrm{CD} 14+$ ) are phagocytosed cells by ADCP (figure 2A-C), whereas CFSE and Zombie Violet double-positive cells (CFSE+/Zombie Violet+) were classified as lysed cells by ADCC (online supplementary figure S2a-c). ADCP and ADCC events are given as a percentage of all CFSEpositive tumor cells.

For the VPA or SAHA-induced secretome experiments, SKBR3 cells were treated with VPA or SAHA, for 24 hours. Afterwards PBMCs were stimulated with the VPA or SAHAinduced secretome (supernatant) of SKBR3 cells for 12 hours, following the ADCP/ADCC assay as described previously.

\section{Analysis of HER2, CALR, CD47 and MCL1 expression in SKBR3 cells}

SKBR3 cells were treated with different VPA and SAHA concentrations as described previously. The cells were further incubated with primary antibodies for HER2, CALR, CD47 or MCL1 (online supplementary table S1) for $30 \mathrm{~min}$ at $4^{\circ} \mathrm{C}$. Unconjugated antibodies were further stained with secondary antibodies for $30 \mathrm{~min}$ at $4^{\circ} \mathrm{C}$ (online supplementary table S1). In the case of an intracellular protein staining, the cells were fixed and permeabilized with the IntraPrep Permeabilizaton Reagent (Beckman Coulter Inc.) according to the manufacturer's protocol. Different protein expression levels were analyzed by the use of a Gallios $\mathrm{G}$ flow cytometer (Beckman Coulter Inc.) and quantified with Kaluza Analysis Software V.2.1.1 (Beckman Coulter Inc.). Histogram overlays were created with FlowJo V.10.6.1 (Becton Dickinson, Franklin Lakes, New Jersey, USA). 


\section{Analysis of $\mathrm{Fc} \gamma \mathrm{R}$ expression on monocytes}

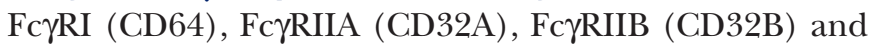
FcyRIII (CD16) expressions on monocytes (CD16+) were measured after the 2.5-hour incubation time of the ADCC/ADCP assay, without prior CFSE labeling of SKBR3 cells and without trastuzumab. As CD32A and CD32B can only be distinguished on their intracellular domain, the cells were fixed and permeabilized with the IntraPrep Permeabilizaton Reagent (Beckman Coulter) according to the manufacturer's protocol. The cells were incubated with primary antibodies (online supplementary table S1) for $30 \mathrm{~min}$ at $4^{\circ} \mathrm{C}$. Unconjugated antibodies were further stained with secondary antibodies for $30 \mathrm{~min}$ at $4^{\circ} \mathrm{C}$ (online supplementary table $\mathrm{S} 1$ ). Fc $\gamma \mathrm{R}$ expression levels were analyzed using a Gallios G flow cytometer (Beckman Coulter Inc.) and quantified with Kaluza Analysis Software V.2.1.1 (Beckman Coulter Inc.). Histogram overlays were created with FlowJo V.10.6.1 (Becton Dickinson, Franklin Lakes, New Jersey, USA).

\section{MCL1 knockdown in SKBR3 cells}

SKBR3 cells $\left(1 \times 10^{6}\right)$ were seeded on 6-well plates. For transfection, the cells were cultured in Opti-MEM Reduced Serum Media (Invitrogen) and the cells were transfected using Lipofectamine 2000 (Invitrogen) according to the manufacturer's protocol. Test groups were transfected with MCL1 small interfering RNA (siRNA; sc-35877; Santa Cruz, Texas, USA), whereas control groups were transfected with AllStars Negative Control siRNA (Quiagen, Hilden, North Rhine-Westphalia, GER). The medium was exchanged to SKBR3 medium 6 hours after transfection. The cells were further cultivated for 24 hours before being used in the ADCP/ADCC assay as described before.

\section{Statistics}

Statistical tests and graphs were performed with GraphPad Prism 7.0a (GraphPad Software, La Jolla, California, USA). The assessment of agreement between FCM and IFC results was done by Bland-Altman plots. ${ }^{31}$ All other activities or expression data are presented by scatter plots (circles, boxes and triangles demonstrate individual values) and corresponding mean (long horizontal line) and SD or SE of the mean (SEM). Comparisons for $\mathrm{ADCP} / \mathrm{ADCC}$ and $\mathrm{Fc} \gamma \mathrm{R}$ results were investigated by a repeated-measures (RM) one-way analysis of variance (ANOVA) and Fisher least significant difference (LSD) post hoc tests. All other comparisons were calculated by a one-way ANOVA and Fisher LSD post hoc tests. Two-sided $p$ values $\leq 0.05$ were regarded as statistically significant.

\section{RESULTS}

\section{ADCP and ADCC measured by FCM detects true phagocytic} events and is comparable with IFC

In order to investigate $\mathrm{ADCP}$ and $\mathrm{ADCC}$, we used an FCM-based approach, which facilitates high throughput data acquisition (online supplementary figure S1). We first determined the reliability of FCM to measure accurate ADCP and ADCC events, in terms of truly phagocytosed/incorporated cells in our setting. In this line, we compared each sample with IFC and conventional FCM, despite considering that both methods rely on different technologies (imaging vs quantification of optical parameters) and different data output. Analysis of ADCP (CFSE+/CD14+) and ADCC (CFSE+/ Zombie Violet+) images show truly phagocytosed and lysed cells, respectively (figure 1a-c; online supplementary figure S3a-c).

Data agreement between the two different methods (IFC vs FCM) was further investigated by Bland-Altman plots. For ADCP, the bias (average of the differences) between IFC and FCM measured values was 0.983 and corresponding $95 \%$ limits of agreement ranged from -2.822 to 4.789 (figure 2d). ADCC comparison resulted in a bias of 0.213 and its associated $95 \%$ limits of agreement from -6.396 to 6.823 (online supplementary figure S3d). We further conducted a Pearson's correlation (scatter plots not shown) for ADCP $(r=0.991 ; \mathrm{p}<0.001)$ and ADCC $(r=0.857 ; \mathrm{p}<0.001)$. Taking together, measurement of ADCP and ADCC by FCM detects true phagocytic events and is comparable with IFC.

\section{Valproic acid and vorinostat enhance trastuzumab-mediated ADCP and trastuzumab-independent cytotoxicity}

In order to investigate a potential additive or synergistic immunomodulatory effect of valproic acid (VPA) or vorinostat (SAHA) on ADCP or ADCC activity, we performed an ADCP/ADCC assay as shown in the online supplementary figure $\mathrm{S} 1$.

We intended to induce limited tumor cell stress that would result in approximately $75 \%$ tumor cell survival. We applied sublethal doses of 5 and $10 \mathrm{mM}$ VPA and 5 and $10 \mu \mathrm{M}$ SAHA, respectively, for 24 hours, which resulted in about $25 \%$ early apoptotic tumor cells (online supplementary figure S2).

Strikingly, trastuzumab-mediated ADCP activity was significantly improved by VPA $(p<0.01$; figure $2 A)$ and SAHA ( $<<0.01$; figure $2 \mathrm{~B}$ ) pretreatment. In contrast, VPA or SAHA alone did not enhance phagocytosis compared with untreated tumor cells (online supplementary figure S4a-b). However, the trastuzumab-enhancing effect was not seen at a lower (12:1) E:T ratio (online supplementary figure S4c-f).

In contrast to ADCP, trastuzumab-independent cytotoxicity was significantly enhanced by VPA $(p<0.05$; figure $3 \mathrm{~A})$ and SAHA $(\mathrm{p}<0.05$; figure $3 \mathrm{~B})$. The addition of trastuzumab however did not affect ADCC compared with untreated tumor cells (online supplementary figure S5a-b). Corresponding to the ADCP results, there was also no enhancing effect observed at an E:T ratio of 12:1 (online supplementary figure S5c-f).

\section{Valproic acid tumor cell treatment increases activating Fc $\gamma R$ IIIA expression on monocytes}

To examine mechanistic effects of VPA or SAHA tumor cell pretreatment on trastuzumab-enhanced ADCP activity, we first investigated potential expression changes 

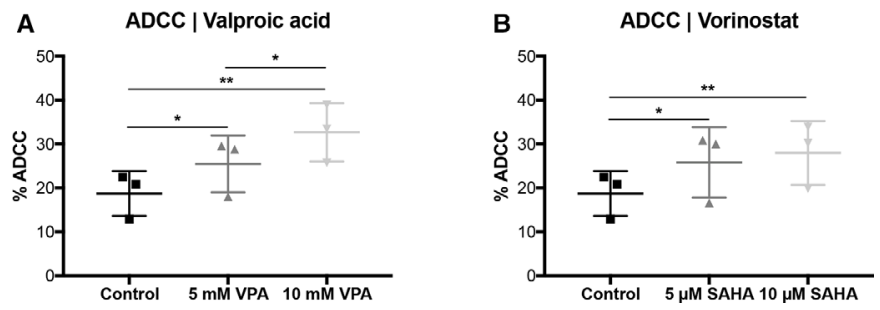

Figure 3 Valproic acid and vorinostat enhance trastuzumabindependent cytotoxicity. SKBR3 cells were treated with VPA or SAHA, for 24 hours, following the ADCP/ADCC as described in figure 1 and measured by FCM. (A) ADCP | valproic acid (B) ADCP | vorinostat. Results are illustrated by scatter plots. Circles, boxes and triangles illustrate individual measured values. \% ADCC is depicted on the ordinate, different treatments and corresponding concentrations are indicated on the abscissa. Mean \% ADCC is illustrated by a long horizontal line \pm SD by short horizontal lines connected with a vertical line. Differences were calculated by repeated-measure one-way ANOVA and Fisher LSD post hoc test. ${ }^{* *} \mathrm{p}<0.01 ;{ }^{* * *} \mathrm{p}<0.001$; ADCC, antibody-dependent cell-mediated cytotoxicity; ADCP, antibody-dependent cellmediated phagocytosis; ANOVA, analysis of variance; FCM, flow cytometry; LSD, least significantdifference; SAHA, suberanilohydroxamic acid; VPA, valproic acid.

of our target receptor HER2. Interestingly, VPA and SAHA lead to a decreased protein expression level compared with untreated tumor cells $(\mathrm{p}<0.05$; online supplementary figure S6a-b).

Next, we examined the different antibody-meditating receptors on monocytes (CD14+). We performed the ADCP/ADCG assay as described above and stained for Fc $\gamma$ RI (CD64), Fc $\gamma$ RIIA (CD32A), Fc $\gamma$ RIIB (CD32B) and FcyRIII (CD16) expression on CD14+cells (figure 4A-D). Protein expression analysis using FCM revealed a statistically significant upregulation of the activating Fc $\gamma$ RIIA by VPA ( $<<0.01$; figure $4 \mathrm{~B})$. All other Fc $\gamma \mathrm{R}$ were not affected by VPA. SAHA had no impact on any Fc $\gamma$ R.

\section{Valproic acid and vorinostat decrease anti-apoptotic molecule MCL1 in tumor cells}

To get a deeper understanding of how VPA and SAHA affect ADCP activity, we examined the major antiapoptotic molecule MCL1. MCL1 protein expression was significantly downregulated by VPA and SAHA $(\mathrm{p}<0.001$; $\mathrm{p}<0.001$; figure $5 \mathrm{~A}$ ). To investigate whether this effect is MCL1 dependent, we knocked-down MCL1 by siRNA (figure 5B) and repeated the ADCP/ADCC assay in the absence of HDACi pretreatment (figure 5C). Strikingly, ADCP activity was significantly enhanced in comparison with untreated controls $(\mathrm{p}<0.05)$. Notably, this effect was only seen in combination with the antibody trastuzumab (online supplementary figure S7a). However, ADCC was not affected by MCL1 knockdown, neither with nor without trastuzumab (online supplementary figure S7b, c).
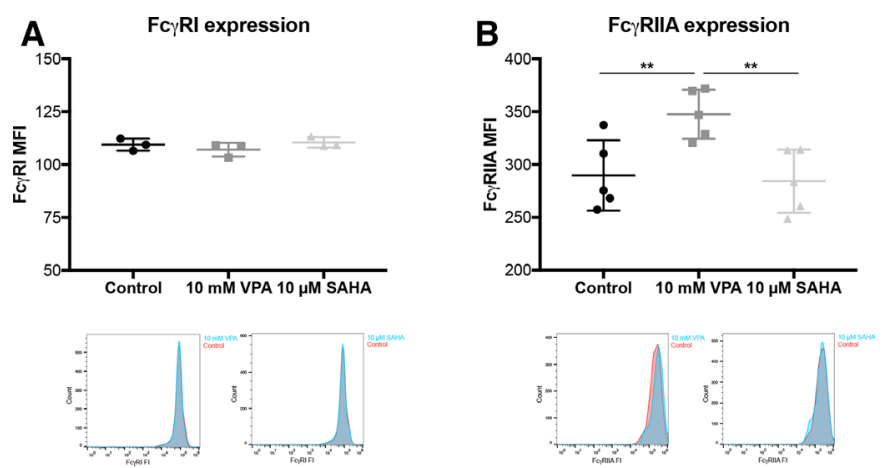

C

FC $\gamma$ RIIB expression

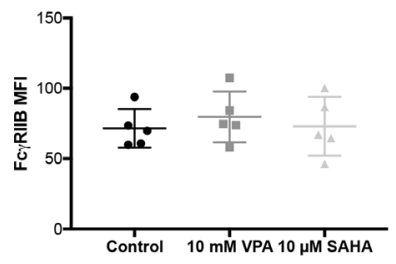

D

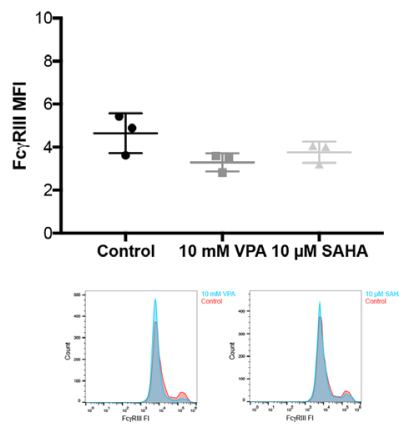

Figure 4 VPA increases expression of the activating Fc $\gamma R$ IIIA on monocytes. SKBR3 cells were treated with VPA or SAHA, for 24 hours, without CFSE labeling of SKBR3 cells and

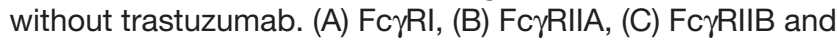
(D) Fc $\gamma R$ III expressions were investigated on monocytes (CD14+) after the 2.5-hour incubation time of the ADCP/ ADCC assay and measured by FCM. Results are illustrated by scatter plots. Circles, boxes and triangles illustrate individual measured values. Each Fc $\gamma \mathrm{R} \mathrm{MFI}$ is depicted on the ordinate, different treatments and corresponding concentrations are indicated on the abscissa. Mean MFI for each marker is illustrated by a long horizontal line \pm SD by short horizontal lines connected with a vertical line.

Corresponding overlay histograms are illustrated. Differences were calculated by repeated-measures one-way ANOVA and Fisher LSD post hoc test. ${ }^{* *} \mathrm{p}<0.01$; CFSE, carboxyfluorescein diacetate succinimidyl ester; Fc $\gamma \mathrm{R}$, Fc gamma receptor; FI, fluorescence intensity; MFI, mean fluorescence intensity; SAHA, suberanilohydroxamic acid; VPA, valproic acid.

\section{Valproic acid and vorinostat induce an immunogenic cell} death (ICD) and downregulates anti-phagocytic CD47 in tumor cells

As VPA and SAHA enhance trastuzumab-independent cytotoxicity, we further investigated if this observation might be explained by the induction of a so-called ICD, which is in part characterized by the surface exposure of CALR, which is counterbalanced by the "do not eat me" signal CD47. ${ }^{27} 32$

FCM analysis revealed a statistically significant upregulation of CALR surface exposure by VPA $(p<0.001$; figure $6 \mathrm{~A})$ and SAHA $(\mathrm{p}<0.05$; figure $6 \mathrm{~B})$, respectively. Moreover, the "do not eat me" signal CD47 was also downregulated on SKBR3 cells by both HDACi, VPA and SAHA $(\mathrm{p}<0.001$; figure 6C; $\mathrm{p}<0.001$, figure 6D). 

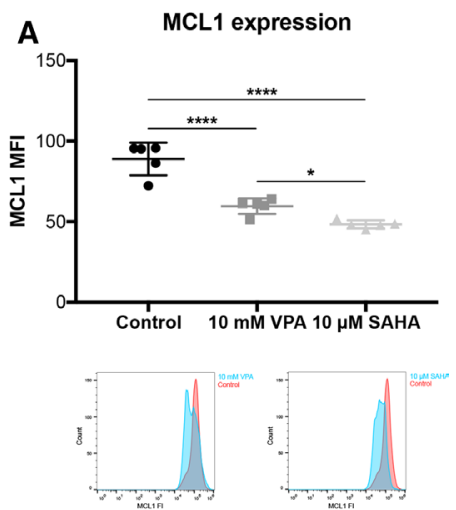

B MCL1 knockdown
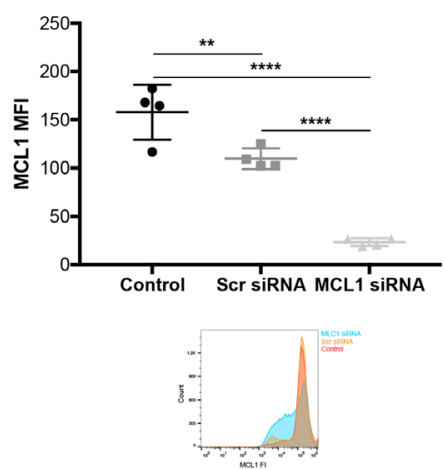

C $\quad A D C P \mid$ Tras + MCL1 knockdown

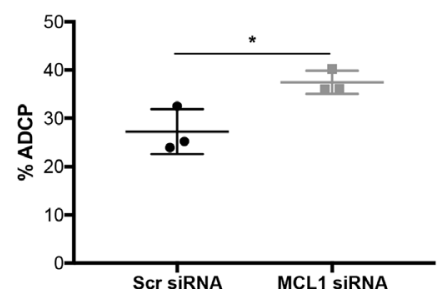

Figure 5 VPA downregulates the anti-apoptotic protein MCL1 in tumor cells. SKBR3 cells were treated with VPA or SAHA, for 24 hours. (A) MCL1 expression determined by FCM. corresponding overlay histograms are illustrated.

(B) MCL1 expression after MCL1 knockdown. MCL1 expression levels were determined by FCM. Corresponding overlay histograms are illustrated. (C) \% ADCP after MCL1 knockdown with trastuzumab in SKBR3 cells. ADCP/ ADCC assay was performed as described in figure 1 and measured by FCM. Results are illustrated by scatter plots. Circles, boxes and triangles illustrate individual measured values. MCL1 MFI and \% ADCP are depicted on the ordinate, different treatments and corresponding concentrations are indicated on the abscissa. Mean MCL1 MFI and mean \% ADCP are illustrated by a long horizontal line $\pm S D$ by short horizontal lines connected with a vertical line. Differences were calculated by a one-way ANOVA and Fisher LSD post hoc test and unpaired Student t-test. ${ }^{*} \mathrm{p}<0.05 ;{ }^{* *} \mathrm{p}<0.01 ;{ }^{* \star \star *} \mathrm{p}<0.0001$; ADCP, antibody-dependent cell-mediated phagocytosis; ANOVA, analysis of variance; FCM, flow cytometry; FI, fluorescence intensity; LSD, least significantdifference; MCL1, myeloid leukemia cell differentiation protein 1; MFI, mean fluorescence intensity; SAHA, suberanilohydroxamic acid; SCR: scrambled; siRNA, small interfering RNA, Tras: trastuzumab; VPA, valproic acid.
A CALR expression | Valproic acid

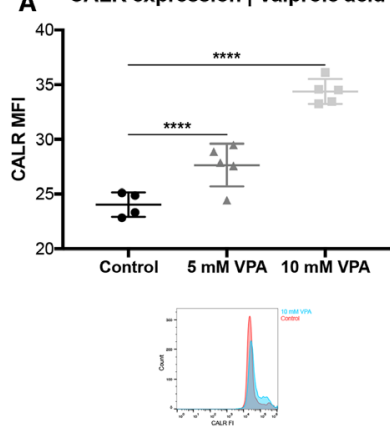

C CD47 expression | Valproic acid

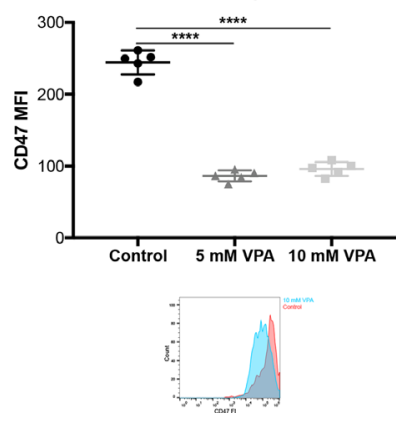

Figure 6 VPA and vorinostat induce an ICD and downregulates anti-phagocytic CD47 in tumor cells. SKBR3 cells were treated with VPA or SAHA, for 24 hours. (A, B) Calreticulin (CALR) and (C, D) CD47 expression levels were determined by FCM. Results are illustrated by scatter plots. Circles, boxes and triangles illustrate individual measured values. MCL1 and CD47 MFI are depicted on the ordinate, different treatments and corresponding concentrations are indicated on the abscissa. Mean CALR and CD47 MFI are illustrated by a long horizontal line \pm SD by short horizontal lines connected with a vertical line. Corresponding overlay histograms are illustrated. Differences were calculated by a one-way ANOVA and Fisher's LSD post hoc test. ${ }^{* *} \mathrm{p}<0.01$; ${ }^{* * * *} p<0.0001$; ANOVA, one-way analysisof variance; CALR, calreticulin; CD47, clusters of differentiation 47; FCM, flow cytometry; FI, fluorescence intensity; ICD, immunogenic cell death; LSD, least significantdifference; MCL1, myeloid leukaemia cell differentiation protein1; MFI, mean fluorescenceintensity; SAHA, suberanilohydroxamic acid; VPA, valproic acid.

Next, we investigated the potential immunostimulatory effects of the HDACi-induced secretome. We stimulated effector cells (PBMCs) with VPA or SAHA-induced secretome of tumor cells for 12 hours prior to the ADCP/ADCC assay. However, neither ADCP nor ADCC, with or without trastuzumab showed enhanced ADCP/ADCC activity compared the tumor cell secretome of untreated cells (online supplementary figure S8a-h).

\section{DISCUSSION}

Recent data provided evidence that epigenetic remodeling by the use of HDACi in combination with other targeted therapies, such as the mAb trastuzumab, is capable of engaging and enhancing an effective anti-tumor immune response. ${ }^{1516}$ However, apart of safety, tolerability and 
the effectiveness of trastuzumab-combined HDACi treatments in vivo, its effect on the immune system, especially on a functional level, remain to be elucidated..$^{33}$ In this study, we investigated the influence of the Food and Drug Administration-approved HDACi, VPA and SAHA on PBMCs effector function in vitro. By the use of a functional immune cell approach, we could demonstrate that both VPA and SAHA enhanced trastuzumab-mediated ADCP activity. In contrast, VPA or SAHA had no effect on the trastuzumab-independent phagocytic killing of tumor cells. This finding might not be very surprising as monocytes are in generally incapable of distinguishing between self and non-self without toll-like receptors, complement or antibody mediation. As the overall functional read-out (ADCP) was increased, we did further investigations in a systematic approach. First, we explored potential changes in trastuzumab's target (HER2). Although the expression of HER2 on target cells is essential for the binding of trastuzumab, the level of HER2 positivity does not influence the extent of ADCP, as both HDACi decreased HER2 surface expression. This is in line with data gained in human studies, which demonstrates that if HER2 is overexpressed, defined by immunohistochemistry staining degree of $3+$ or a HER2/chromosome 17 fluorescence in situ hybridisation ratio of $\geq 2.0$, then the degree of positivity of HER2 expression has no major influence on the clinical outcome. ${ }^{34-37}$ Correspondingly, another study revealed that HER2-non-amplified cancer cell lines are still highly susceptible to trastuzumab-mediated cell killing, whereas HER2-non-expressing cell lines are not. ${ }^{38}$ However, the extent of response does not necessarily depend on overall HER2 expression.

We used an FCM-based evaluation for ADCC and ADCP activity, a technique that is also suitable for clinical analysis of larg patient cohorts. ${ }^{30}$ A potential uncertainty of this technique is false-positive ADCP events, in terms of true engulfed tumor cells by monocytes. In contrast, IFC provides high-resolution images at the single-cell level, which is particularly advantageous when analyzing cellular interactions, such as ADCP activity in vitro. However, this technique is time-consuming and may be impractical when measuring large sample sizes. The comparability and reliability of FCM in comparison with IFC, in terms of true ADCP events, have not been assessed yet. Therefore, we adapted the conventional FCM ADCP/ADCC assays for an IFC device..$^{28-30} 39$ We demonstrate that conventional FCM detects true phagocytic and cytotoxic singlecell events and is comparable with IFC. Taking this matter of fact into account, as well as the more practicability of FCM compared with IFC, we kept using the conventional FCM approach for our ADCP/ADCC assay.

As the level of HER2 positivity has no influence on ADCP activity, we next investigated potential changes and influence on trastuzumab's effector side $(\mathrm{Fc} \gamma \mathrm{R})$. In this line, murine data demonstrated the importance of the interaction between Fc $\gamma$ Rs and trastuzumab for an optimal anti-tumor immune response in vivo. ${ }^{640}$ Fc $\gamma$ RIIA (CD32A) plays an essential role in the mediation of an effective ADCP activity. Strikingly, VPA treatment upregulates FcyRIIA expression on monocytes (CD14+), whereas SAHA had no effect on any Fc $\gamma \mathrm{R}$. Accordingly, antibody engineering with enhanced binding affinity to FcyRIIA has been shown to increase macrophagemediated phagocytosis. ${ }^{41}{ }^{42}$ These data highlight a potential synergistic effect of antibodies with optimized binding affinity and $\mathrm{Fc} \gamma \mathrm{R}$ upregulation by epigenetic remolding of VPA.

In contrast to ADCP, ADCC seems to be unaffected by any HDACi treatment. This finding could be explained by the lack of Fc $\gamma$ RIII (CD16) upregulation, which is the main antibody-mediating receptor of NK cells. Strikingly, trastuzumab-independent cytotoxicity is significantly enhanced by both HDACi, VPA and SAHA.

As resistance to spontaneous or therapy-induced cell death is as major hallmark of cancer ${ }^{43}$ we further examined a possible effect on cancer cell-intrinsic resistance to immune cell-mediated cell death. The so-called apoptotic trigger is meticulously controlled by proapoptotic and anti-apoptotic proteins. Tumor cells can circumvent cell death by increasing the expression of anti-apoptotic regulators, such as MCL1. Therefore, we investigated potential counterbalancing effects of VPA and SAHA on MCL1 protein expression. Tumor cell treatment with VPA or SAHA resulted in a downregulation of MCL1 protein expression. To further investigate whether the enhanced ADCP effect of VPA is MCL1 dependent, we knocked-down MCL1 by the use of siRNA. MCL1 knockdown alone without any HDACi treatment resulted in an enhanced ADCP activity. Along this line, trastuzumab itself seems to have an intrinsic ability to reduce MCL1 expression. ${ }^{44}$ The inhibition of anti-apoptotic proteins, such as MCL1, might be especially effective in cancer cells with rat sarcoma mutations as they have an upregulated anti-apoptotic signaling cascade. ${ }^{24}$

Due to the enhanced trastuzumab-independent cytotoxicity, we further investigated the potential induction of immunogenic cell death (CALR) and its counterplayers (CD47) as a "do not eat me" signal. Both HDACi treatments resulted in a cell surface exposure of CALR, which serves as a surrogate marker for an endoplasmic reticulum (ER) stress response. At the same time, the CALR counterbalancing "do not eat me" signal CD47 has been also downregulated. This finding underlines the importance of a rational combined treatment approach with immunological stimulatory properties, as CD47 is a major inhibitor of an ICD-induced effective anti-tumor immune response. ${ }^{45}{ }^{46}$ Moreover, VPA and SAHA might be of major interest when combined with other targeted therapies or immune-checkpoint-blocking antibodies. ${ }^{47} 48$

As the induction of an ICD is often linked to the secretion of a variety of immunostimulatory signals, we assessed the potential effects of the HDACi-induced secretome on a functional level. Surprisingly, in the HDACi-induced secretome of cancer cells showed no enhancing effect on neither ADCP nor ADCC activity underscoring the relevance of drug-induced direct modulation of cell signaling. 
It should be noted that the two different HDACi used in the present study (SAHA and VPA) act on different HDACs enzymes. In particular, SAHA inhibits HDACs $1,2,3,4,5,6,7,8,9$ and $10{ }^{49}$ whereas VPA acts only on HDACs $1,2,3,4,5$ and $7 .^{50}$ We suggest that this is the reason for the differential treatment effects, such as enhanced Fc $\gamma$ RIIA expression, which were only seen for VPA but not SAHA. On the other hand, SAHA downregulated the anti-apoptotic protein MCL1 more potently compared with VPA $(p<0.05)$, which might serve as an explanation why the overall ADCP activity still gets statistically significantly upregulated by SAHA, besides the lack of FcyRIIA upregulation.

In conclusion, the immunomodulatory activities of HDACi (VPA and SAHA) support a rationale combined treatment approach with trastuzumab for breast cancer treatment. Our data provide a rational concept to design a clinical study, which combines HADCi and anti-HER2 antibodies. Certainly, such a study might not be restricted to low-risk breast cancer patients eligible for anti-HER2 monotherapy (trastuzumab) but might also include highrisk breast cancer patient suffering from nodal-positive $(\mathrm{N}+)$ and estrogen receptor-negative (ER-) tumors, who are currently advised to receive a dual anti-HER2 treatment (trastuzumab and pertuzumab). ${ }^{51}$

\section{CONCLUSIONS}

Our data suggest that the immune system plays a key role in the mechanism underlying HDACi-mediated ADCP enhancement. The enhanced trastuzumab-mediated phagocytosis and trastuzumab-independent cytotoxicity is a result of the multimodal HDACi treatment effect, which induces a bona fide ICD, enhances susceptibility to phagocytosis and apoptosis, as well as increases antibodymediating receptor expression.

Acknowledgements The authors would like to thank Andreas Spittler (Core Facility Flow Cytometry, Medical University of Vienna, Vienna, Austria) for his expert help with the Imaging Flow Cytometry.

Contributors JL and MB: the study. LH, JK, JH and SP: performed the experiments. GE: provided experimental resources and knowledge. JL: calculated the statistics; drew the data figures and tables; wrote the manuscript. JL, JK and MB: interpreted the results. LH and JK: drew the vector graphics. JL, LH JK, GE and MB: edited the manuscript. JL and MB: supervised the study. All authors approved the latest version of the manuscript.

Funding This study was supported in part by research funds of Medical University of Vienna, as well as a personal research fund of the "Fellinger Cancer Research" (granted to JL).

Competing interests None declared.

Patient consent for publication Not required.

Ethics approval This study was carried out in consensus with "Good Scientific Practice Guidelines" of the Medical University of Vienna, as well as the latest "Declaration of Helsinki". The study protocol was reviewed and approved by the "Ethics Committee" of the Medical University of Vienna (\#1374/2014).

Provenance and peer review Not commissioned; externally peer reviewed.

Data availability statement All data relevant to the study are included in the article or uploaded as supplementary information.

Open access This is an open access article distributed in accordance with the Creative Commons Attribution Non Commercial (CC BY-NC 4.0) license, which permits others to distribute, remix, adapt, build upon this work non-commercially, and license their derivative works on different terms, provided the original work is properly cited, appropriate credit is given, any changes made indicated, and the use is non-commercial. See http://creativecommons.org/licenses/by-nc/4.0/.

\section{ORCID iDs}

Johannes Laengle http://orcid.org/0000-0003-4771-1398

Gerda Egger http://orcid.org/0000-0003-2489-155X

Michael Bergmann http://orcid.org/0000-0001-8529-1166

\section{REFERENCES}

1 Slamon D, Eiermann W, Robert N, et al. Adjuvant trastuzumab in HER2-positive breast cancer. N Engl J Med 2011;365:1273-83.

2 Senkus E, Kyriakides S, Ohno S, et al. Primary breast cancer: ESMO clinical practice guidelines for diagnosis, treatment and follow-up. Ann Oncol 2015;26 Suppl 5:v8-30.

3 Vu T, Claret FX. Trastuzumab: updated mechanisms of action and resistance in breast cancer. Front Oncol 2012;2:62.

4 Spector NL, Blackwell KL. Understanding the mechanisms behind trastuzumab therapy for human epidermal growth factor receptor 2-positive breast cancer. J Clin Oncol 2009;27:5838-47.

5 Mir O, Berveiller P, Pons G. Trastuzumab--mechanism of action and use. N Engl J Med 2007;357:1664-5. author reply 1665-6.

6 Clynes RA, Towers TL, Presta LG, et al. Inhibitory Fc receptors modulate in vivo cytotoxicity against tumor targets. Nat Med 2000;6:443-6.

7 Shi Y, Fan X, Deng H, et al. Trastuzumab triggers phagocytic killing of high HER2 cancer cells in vitro and in vivo by interaction with Fc $\gamma$ receptors on macrophages. J Immunol 2015;194:4379-86.

8 Baselga J, Carbonell X, Castañeda-Soto N-J, et al. Phase II study of efficacy, safety, and pharmacokinetics of trastuzumab monotherapy administered on a 3-weekly schedule. J Clin Oncol 2005;23:2162-71.

9 Vogel CL, Cobleigh MA, Tripathy D, et al. Efficacy and safety of trastuzumab as a single agent in first-line treatment of HER2-overexpressing metastatic breast cancer. J Clin Oncol 2002;20:719-26.

10 West AC, Johnstone RW. New and emerging HDAC inhibitors for cancer treatment. J Clin Invest 2014;124:30-9.

11 Jones PA, Ohtani $\mathrm{H}$, Chakravarthy A, et al. Epigenetic therapy in immune-oncology. Nat Rev Cancer 2019;19:151-61.

12 Bali P, Pranpat M, Swaby R, et al. Activity of suberoylanilide hydroxamic acid against human breast cancer cells with amplification of HER-2. Clin Cancer Res 2005;11:6382-9.

13 Bolden JE, Shi W, Jankowski K, et al. HDAC inhibitors induce tumorcell-selective pro-apoptotic transcriptional responses. Cell Death Dis 2013;4:e519.

14 Göttlicher M, Minucci S, Zhu P, et al. Valproic acid defines a novel class of HDAC inhibitors inducing differentiation of transformed cells. Embo J 2001;20:6969-78.

15 Medon M, Vidacs E, Vervoort SJ, et al. HDAC Inhibitor Panobinostat Engages Host Innate Immune Defenses to Promote the Tumoricidal Effects of Trastuzumab in HER2 ${ }^{+}$Tumors. Cancer Res 2017;77:2594-606

16 West AC, Mattarollo SR, Shortt J, et al. An intact immune system is required for the anticancer activities of histone deacetylase inhibitors. Cancer Res 2013;73:7265-76.

17 Armeanu S, Bitzer M, Lauer UM, et al. Natural killer cell-mediated Iysis of hepatoma cells via specific induction of NKG2D ligands by the histone deacetylase inhibitor sodium valproate. Cancer Res 2005;65:6321-9.

18 Christiansen AJ, West A, Banks K-M, et al. Eradication of solid tumors using histone deacetylase inhibitors combined with immunestimulating antibodies. Proc Natl Acad Sci U S A 2011;108:4141-6.

19 Skov S, Pedersen MT, Andresen L, et al. Cancer cells become susceptible to natural killer cell killing after exposure to histone deacetylase inhibitors due to glycogen synthase kinase-3-dependent expression of MHC class I-related chain A and B. Cancer Res 2005;65:11136-45

20 Chen W, Wei F, Xu J, et al. Trastuzumab enhances the anti-tumor effects of the histone deacetylase inhibitor sodium butyrate on a HER2-overexpressing breast cancer cell line. Int $\mathrm{J} \mathrm{Mol}$ Med 2011;28:985-91.

21 Huang X, Wang S, Lee C-K, et al. HDAC inhibitor SNDX-275 enhances efficacy of trastuzumab in erbB2-overexpressing breast cancer cells and exhibits potential to overcome trastuzumab resistance. Cancer Lett 2011;307:72-9.

22 Fuino L, Bali P, Wittmann S, et al. Histone deacetylase inhibitor LAQ824 down-regulates HER-2 and sensitizes human breast cancer 
cells to trastuzumab, taxotere, gemcitabine, and epothilone B. Mol Cancer Ther 2003;2:971-84.

23 Goldstein LJ, Zhao F, Wang M, et al. A phase I/II study of suberoylanilide hydroxamic acid (SAHA) in combination with trastuzumab (Herceptin) in patients with advanced metastatic and/or local chest wall recurrent HER2-amplified breast cancer: a trial of the ECOG-ACRIN cancer research Group (E1104). Breast Cancer Res Treat 2017;165:375-82.

24 Kasper S, Breitenbuecher F, Reis $\mathrm{H}$, et al. Oncogenic Ras simultaneously protects against anti-EGFR antibody-dependent cellular cytotoxicity and EGFR signaling blockade. Oncogene 2013;32:2873-81.

25 Pincetic A, Bournazos S, DiLillo DJ, et al. Type I and type II Fc receptors regulate innate and adaptive immunity. Nat Immunol 2014:15:707-16.

26 Veillette A, Chen J. SIRP $\alpha$-CD47 immune checkpoint blockade in anticancer therapy. Trends Immunol 2018;39:173-84.

27 Obeid M, Tesniere A, Ghiringhelli F, et al. Calreticulin exposure dictates the immunogenicity of cancer cell death. Nat Med 2007;13:54-61.

28 Bracher M, Gould HJ, Sutton BJ, et al. Three-colour flow cytometric method to measure antibody-dependent tumour cell killing by cytotoxicity and phagocytosis. J Immunol Methods 2007;323:160-71.

29 Karagiannis $\mathrm{P}$, Singer $\mathrm{J}$, Hunt $\mathrm{J}$, et al. Characterisation of an engineered trastuzumab IgE antibody and effector cell mechanisms targeting HER2/neu-positive tumour cells. Cancer Immunol Immunother 2009;58:915-30.

30 Petricevic B, Laengle J, Singer J, et al. Trastuzumab mediates antibody-dependent cell-mediated cytotoxicity and phagocytosis to the same extent in both adjuvant and metastatic HER2/neu breast cancer patients. J Trans/ Med 2013;11:307.

31 Bland JM, Altman DG. Statistical methods for assessing agreement between two methods of clinical measurement. Lancet 1986;1:307-10

32 Chao MP, Jaiswal S, Weissman-Tsukamoto R, et al. Calreticulin is the dominant pro-phagocytic signal on multiple human cancers and is counterbalanced by CD47. Sci Transl Med 2010;2:63ra94.

33 Lim B, Murthy RK, Lee J, et al. A phase lb study of entinostat plus lapatinib with or without trastuzumab in patients with HER2-positive metastatic breast cancer that progressed during trastuzumab treatment. Br J Cancer 2019;120:1105-12.

34 Perez EA, Reinholz MM, Hillman DW, et al. HER2 and chromosome 17 effect on patient outcome in the N9831 adjuvant trastuzumab trial. J Clin Oncol 2010;28:4307-15.

35 Dowsett M, Procter M, McCaskill-Stevens W, et al. Disease-free survival according to degree of HER2 amplification for patients treated with adjuvant chemotherapy with or without 1 year of trastuzumab: the HERA trial. J Clin Oncol 2009;27:2962-9.
36 Zabaglo L, Stoss O, Rüschoff J, et al. HER2 staining intensity in HER2-positive disease: relationship with fish amplification and clinical outcome in the HERA trial of adjuvant trastuzumab. Ann Oncol 2013;24:2761-6.

37 Vilquin P, Donini CF, Villedieu M, et al. Microrna-125B upregulation confers aromatase inhibitor resistance and is a novel marker of poor prognosis in breast cancer. Breast Cancer Res 2015;17.

38 Collins DM, O'Donovan N, McGowan PM, et al. Trastuzumab induces antibody-dependent cell-mediated cytotoxicity (ADCC) in HER-2-non-amplified breast cancer cell lines. Ann Oncol 2012;23:1788-95.

39 Helguera G, Rodríguez JA, Luria-Pérez R, et al. Visualization and quantification of cytotoxicity mediated by antibodies using imaging flow cytometry. J Immunol Methods 2011;368:54-63.

40 Spiridon $\mathrm{Cl}$, Guinn S, Vitetta ES. A comparison of the in and in vivo activities of $\lg G$ and $F\left(a b^{\prime}\right) 2$ fragments of a mixture of three monoclonal anti-Her-2 antibodies. Clin Cancer Res 2004;10:3542-51.

41 Richards JO, Karki S, Lazar GA, et al. Optimization of antibody binding to FcgammaRIIA enhances macrophage phagocytosis of tumor cells. Mol Cancer Ther 2008;7:2517-27.

42 Jung ST, Kelton W, Kang TH, et al. Effective phagocytosis of low HER2 tumor cell lines with engineered, aglycosylated IgG displaying high Fc $\gamma R$ Illa affinity and selectivity. ACS Chem Biol 2013;8:368-75.

43 Hanahan D, Weinberg RA. Hallmarks of cancer: the next generation. Cell 2011;144:646-74.

44 Henson ES, Hu X, Gibson SB. Herceptin sensitizes ErbB2overexpressing cells to apoptosis by reducing antiapoptotic $\mathrm{Mcl}-1$ expression. Clin Cancer Res 2006;12:845-53.

45 Tseng D, Volkmer J-P, Willingham SB, et al. Anti-CD47 antibodymediated phagocytosis of cancer by macrophages primes an effective antitumor T-cell response. Proc Natl Acad Sci U S A 2013;110:11103-8

46 Liu X, Pu Y, Cron K, et al. CD47 blockade triggers T cell-mediated destruction of immunogenic tumors. Nat Med 2015;21:1209-15.

47 Su S, Zhao J, Xing Y, et al. Immune checkpoint inhibition overcomes ADCP-Induced immunosuppression by macrophages. Cell 2018:175:442-57. e23.

48 Gordon SR, Maute RL, Dulken BW, et al. PD-1 expression by tumourassociated macrophages inhibits phagocytosis and tumour immunity. Nature 2017:545:495-9.

49 Marks PA, Breslow R. Dimethyl sulfoxide to vorinostat: development of this histone deacetylase inhibitor as an anticancer drug. Nat Biotechnol 2007;25:84-90

50 Gurvich N, Tsygankova OM, Meinkoth JL, et al. Histone deacetylase is a target of valproic acid-mediated cellular differentiation. Cancer Res 2004;64:1079-86.

51 von Minckwitz G, Procter M, de Azambuja E, et al. Adjuvant pertuzumab and trastuzumab in early HER2-positive breast cancer. $N$ Engl J Med 2017;377:122-31. 